\title{
Flamelet-based RANS Computations of Supersonic Reacting Flows in a Model Scramjet Combustor
}

\author{
Nicholas Arnold-Medabalimi*, Karthik Duraismay ${ }^{\dagger}$ \\ Department of Aerospace Engineering, \\ University of Michigan, Ann Arbor, Michigan 48109.
}

\begin{abstract}
The turbulent, reacting flow field of a Scramjet combustor is investigated using flameletbased Reynolds Averaged Navier-Stokes computations. The simulations target the model combustor configuration of Gamba et al. (2011, 2012), which includes a single injector, an inlet-induced shock train and shock/boundary layer/combustion interactions. Multiple fuel injection rates are considered and the impact of the equivalence ratio on the flow structure, heat release and flame properties is examined. The effect of the shock system on the reacting layers and heat release is examined. The computed results are in excellent qualitative agreement with Schlieren, Planar Laser-induced fluorescence imagery and chemiluminescence results. Comparisons with pressure measurements again show a good degree of correlation apart from a small constant offset.
\end{abstract}

\section{Introduction}

The development of the next generation of high speed air-breathing vehicles hinges on the design of new high enthalpy propulsion systems. Potential applications include passenger transport, reusable launch vehicles for both space launch and munitions delivery. As a consequence of their mechanical simplicity, Scramjet vehicles have demonstrated promise as a viable choice. Major Scramjet programs include the National Aerospace Plane, the NASA X-43, the Air Force X-51, and the University of Queensland's HyShot. These vehicles rely on a close system integration of the airframe and propulsion system. A majority of mature designs require use of the forward fuselage as a compression surface for the propulsion systems. In the combustor, fuel is typically injected as a transverse jet or through a wedge flame-holder. A key challenge is that the fuel has to be mixed and burnt during its short residence time $(O(1 \mathrm{~ms}))$ in the combustor. Additional complexities are introduced via a wide variety of fluid dynamic phenomena including complicated interactions of shock waves and turbulent boundary layers with mixing and combustion.

While large eddy simulations (LES) are trustworthy in representing processes such as turbulent mixing and flow separation, these techniques continue to be infeasible (unless a near-wall model is incorporated ${ }^{9}$ ) even to analyze a single flight condition. Reynolds Averaged Navier-Stokes (RANS) models, on the other hand, are multiple orders-of-magnitude more affordable than even wall-modeled LES and will thus continue to play a key role in various stages of the design process for at least the next few decades. Assessing the reliability of RANS tools in such complex flows is one of the main goals of this work. It is especially critical for these models to represent, to an appropriate level of fidelity, supersonic turbulent combustion and the associated heat release in the presence of shocks. It is well-recognized ${ }^{22}$ that coherent vortical structures have a significant effect on the reaction rates. Further, the ability of RANS-based combustion models to represent the interaction of shocks with reaction layers is not well-quantified. At a fundamental level, the loss of information in the ensemble averaging process raises open questions about the applicability of RANS models in the above situations. In a practical setting, however, RANS has been shown to predict the overall performance and major flow processes $5,10,14,20,21$ to an acceptable quality in Scramjet problems under steady operating conditions.

Accurately representing the interaction of turbulence and combustion and the associated impact on the heat release is of critical importance to the success of RANS models. In this setting, practical combustion

\footnotetext{
* Graduate Student, AIAA Student Member

${ }^{\dagger}$ Assistant Professor, AIAA Member
} 
models rely on reduced chemical pathways and the computed transport of chemical species which requires closure for the chemical source term. A very wide range of techniques (for instance, Refs. 1-3,11,15) have been proposed to address this issue. Extending many of these methods (for instance, through assumed or transported probability density function ${ }^{4}$ approaches) to include even a reduced set of species leads to typically high computational costs.

In this work, we will instead pursue a flamelet-based approach ${ }^{18}$ which assumes that chemical time scales are fundamentally shorter than turbulent scales. This allows the flame to be modeled as locally onedimensional, and thus for the combustion process to be pre-computed and tabulated as a function of a few variables. This can significantly reduce computational time as the combustion kinetics can be interpolated as required by the solver during run-time. In the context of flows directly relevant to this work, Saghafian et al. ${ }^{20}$ have applied this technique to a supersonic jet-in-crossflow (JICF) and Terrapon et al. ${ }^{21}$ have extended it to the Hyshot combustor.

In this work, we assess the models in the context of the simplified model Scramjet combustor of Gamba et al. ${ }^{7,8}$ The configuration includes a single injector, an inlet-induced shock train and shock/boundary layer/combustion interactions and thus represents an intermediate level of complexity (between the JICF and the full engine). High resolution PLIF, Schlieren and pressure measurements are available over a range of operating conditions. The main aim of his work to examine the key features of the reaction layer, flame structures and their interactions with the inlet and injector-induced shock systems. This work is of a preliminary nature and sets the stage for a deeper investigation into quantifying uncertainties in RANS simulations of Scramjet problems and to develop effective reduced-order models.

\section{Computational Methodology}

The governing equations for the density $(\bar{\rho})$, momentum $\left(\bar{\rho} \tilde{u}_{i}\right)$ and energy $\left(\bar{\rho} \tilde{e}_{t}\right)$ are represented by the Favre-Averaged Navier-Stokes equations: ${ }^{24}$

$$
\begin{aligned}
\frac{\partial \bar{\rho}}{\partial t}+\frac{\partial}{\partial x_{j}}\left(\bar{\rho} \tilde{u}_{j}\right) & =0 \\
\frac{\partial \bar{\rho} \tilde{u}_{i}}{\partial t}+\frac{\partial}{\partial x_{j}}\left(\bar{\rho} \tilde{u}_{i} \tilde{u}_{j}\right) & =-\frac{\partial \bar{p}}{\partial x_{i}}+\frac{\partial}{\partial x_{j}}\left(\bar{\sigma}_{i j}+\tau_{i j}\right) \\
\frac{\partial \bar{\rho} \tilde{e}_{t}}{\partial t}+\frac{\partial}{\partial x_{j}}\left(\bar{\rho} \tilde{u}_{j} \tilde{e}_{t}\right) & =\frac{\partial}{\partial x_{j}}\left[\left(\frac{\lambda}{c_{p}}+\frac{\mu_{t}}{P r_{t}}\right) \frac{\partial \tilde{h}}{\partial x_{j}}+\left(\mu+\frac{\mu_{t}}{S c_{k}}\right) \frac{\partial k}{\partial x_{j}}\right]-\frac{\partial\left(\tilde{u}_{j} \bar{p}\right)}{\partial x_{j}}+\frac{\partial}{\partial x_{j}}\left[\tilde{u}_{i}\left(\bar{\sigma}_{i j}+\tau_{i j}\right)\right],
\end{aligned}
$$

where $\bar{\sigma}_{i j}$ and $\tau_{i j}$ represent the molecular and turbulent stresses, respectively. A Reynolds analogy for turbulent heat flux and a Boussinesq approximation for the turbulent transport is additionally assumed. These equations have been derived at the unity Lewis number condition. $\operatorname{Pr}_{t}$ is the turbulent Prandtl Number and $S c_{()}$is the Schmidt number.

Turbulence is modeled using the Menter SST model ${ }^{12}$ which adds two additional transport variables representing turbulent kinetic energy $(k)$ and the specific dissipation $(\omega)$ representing the scale of the turbulence. These equations are given by:

$$
\begin{aligned}
& \frac{\partial}{\partial t}(\bar{\rho} k)+\frac{\partial}{\partial x_{j}}(\bar{\rho} \tilde{u} k)=P-\beta^{*} \bar{\rho} \omega k+\frac{\partial}{\partial x_{j}}\left[\left(\mu+\sigma_{k} \mu_{t}\right) \frac{\partial k}{\partial x_{j}}\right] \\
& \frac{\partial}{\partial t}(\bar{\rho} \omega)+\frac{\partial}{\partial x_{j}}(\bar{\rho} \tilde{u} \omega)=\frac{\gamma}{\nu_{t}} P-\beta \bar{\rho} \omega^{2}+\frac{\partial}{\partial x_{j}}\left[\left(\mu+\sigma_{\omega} \mu_{t}\right) \frac{\partial \omega}{\partial x_{j}}\right]+2\left(1-F_{1}\right) \frac{\bar{\rho} \sigma_{d}}{\omega} \frac{\partial k}{\partial x_{j}} \frac{\partial \omega}{\partial x_{j}}
\end{aligned}
$$

As mentioned previously, turbulent combustion is modeled using the flamelet concept. Flamelet modeling traditionally has been used in low Mach number flows ${ }^{16}$ but has recently reformulated for higher Mach numbers. ${ }^{20,21}$ Specifically, properties are tabulated using a three-dimensional table based on the reaction progress variable $\tilde{C}$, mixture fraction $\tilde{Z}$, and the variance of the mixture fraction $\tilde{Z}^{\prime \prime 2}$. The transport 
equations for these variables are given by

$$
\begin{aligned}
\frac{\partial}{\partial t}(\bar{\rho} \tilde{Z})+\frac{\partial}{\partial x_{j}}(\bar{\rho} \tilde{u} \tilde{Z}) & =\frac{\partial}{\partial x_{j}}\left[\left(\frac{\lambda}{c_{p}}+\frac{\mu_{t}}{S c_{z}}\right) \frac{\partial \tilde{Z}}{\partial x_{j}}\right] \\
\frac{\partial}{\partial t}\left(\bar{\rho} \tilde{Z}^{\prime \prime 2}\right)+\frac{\partial}{\partial x_{j}}\left(\bar{\rho} \tilde{u} \tilde{Z}^{\prime \prime 2}\right) & =\frac{\partial}{\partial x_{j}}\left[\left(\frac{\lambda}{c_{p}}+\frac{\mu_{t}}{S c_{z^{\prime \prime 2}}}\right) \frac{\partial \tilde{Z}^{\prime \prime 2}}{\partial x_{j}}\right]+2 \frac{\mu_{t}}{S c_{z^{\prime \prime 2}}} \frac{\partial \tilde{Z}}{\partial x_{j}} \frac{\partial \tilde{Z}}{\partial x_{j}}-c_{\chi} \bar{\rho} \tilde{\chi}^{\prime \prime} \\
\frac{\partial}{\partial t}(\bar{\rho} \tilde{C})+\frac{\partial}{\partial x_{j}}(\bar{\rho} \tilde{u} \tilde{C}) & =\frac{\partial}{\partial x_{j}}\left[\left(\frac{\lambda}{c_{p}}+\frac{\mu_{t}}{S c_{c}}\right) \frac{\partial \tilde{C}}{\partial x_{j}}\right]+\tilde{\dot{\omega}}_{C} .
\end{aligned}
$$

To obtain closure, the equations of state, material properties, and the source term are derived using the following simplified approximations, which were first reported by Saghafian et al.. ${ }^{19}$

$$
\begin{aligned}
\hat{T} & =\hat{T}_{0}+\frac{\tilde{\gamma}_{0}-1}{a_{\gamma}}\left(e^{a_{\gamma}\left(\tilde{e}-\tilde{e}_{0}\right) / \tilde{R}_{0}}-1\right) \\
\tilde{e} & =\tilde{e}_{0}+\frac{\tilde{R}_{0}}{a_{\gamma}} \ln \left(1+\frac{a_{\gamma}\left(\hat{T}-\hat{T}_{0}\right)}{\tilde{\gamma}_{0}-1}\right) \\
\tilde{h} & =\tilde{e}+\tilde{R}_{0} \hat{T} \\
\bar{p} & =\bar{\rho} \tilde{R}_{0} \hat{T} \\
\frac{\lambda}{c_{p}} & =\left(\frac{\lambda}{c_{p}}\right)_{0}\left[\frac{\hat{T}}{\hat{T}}\right]^{0.62} \\
\mu & =\mu_{0}\left[\frac{\hat{T}}{\hat{T}_{0}}\right]^{0.7} \\
\gamma & =\tilde{\gamma}_{0}+a_{\gamma}\left(\hat{T}-\hat{T}_{0}\right) \\
a & =\sqrt{\gamma \tilde{R}_{0} \hat{T}}
\end{aligned}
$$

In these equations, the ( $)_{0}$ variables are interpolated from the chemical table (described below). It should be noted that a table is computed for a given reference pressure. To account for deviations of this pressure, the source term in the progress variable transport equation $3 \mathrm{c}$ is scaled as $\tilde{\dot{\omega}}_{C}=\tilde{\dot{\omega}}_{0 C} \frac{\bar{p}^{2}}{p_{r e f}^{2}}$. In addition, the scalar dissipation rate term $\tilde{\chi}^{\prime \prime}$ is approximated as $2 C_{\mu} \omega$.

Thus, the governing equations thus involve the transport of 10 variables

$$
Q=\left\{\bar{\rho}, \bar{\rho} \tilde{u}, \bar{\rho} \tilde{e} t, \bar{\rho} k, \bar{\rho} \omega, \bar{\rho} \tilde{Z}, \bar{\rho} \tilde{Z}^{\prime \prime 2}, \bar{\rho} \tilde{C}\right\}^{T}
$$

which are solved in a fully coupled fashion ${ }^{5}$ using an implicit Euler time-integration with local time-stepping. The governing equations are spatially discretized using a cell-centered finite volume scheme on polyhedral unstructured mesh..$^{5,21}$ The Barth-Jesperson/Venkatakrishnan method ${ }^{23}$ is used to limit the solution gradients and the inviscid fluxes are computed using the HLLC Riemann solver that operates on all ten conserved variables. The same numerical discretization is used for the mean flow quantities and the transported scalars. The solution gradients are evaluated at the cell centers are then averaged at cell faces to compute the diffusive fluxes. ${ }^{13}$

\section{A. Chemical properties}

The chemical table was generated using the FlameMaster suite. ${ }^{17}$ The steady flamelet solver was used to generate a 3D linear Cartesian table with the dimensions of mean mixture fraction, variance of the mixture fraction and the mean progress variable. In the present work, the combustion reaction involves pure oxygen and hydrogen, and thus the mass fraction of $\mathrm{H}_{2} \mathrm{O}$ is taken to be the progress variable. The boundary conditions used were $1593 \mathrm{~K}$ for the oxidizer side and $300 \mathrm{~K}$ for the fuel side. The background reference pressure was selected to be $105 \mathrm{kPa}$ based on the mean pressure distribution. Individual flamelets were generated for a range of scalar dissipation rates along the upper branch of the S-curve and then downselected to maintain monotonicity and prevent flamelets from intersecting. 


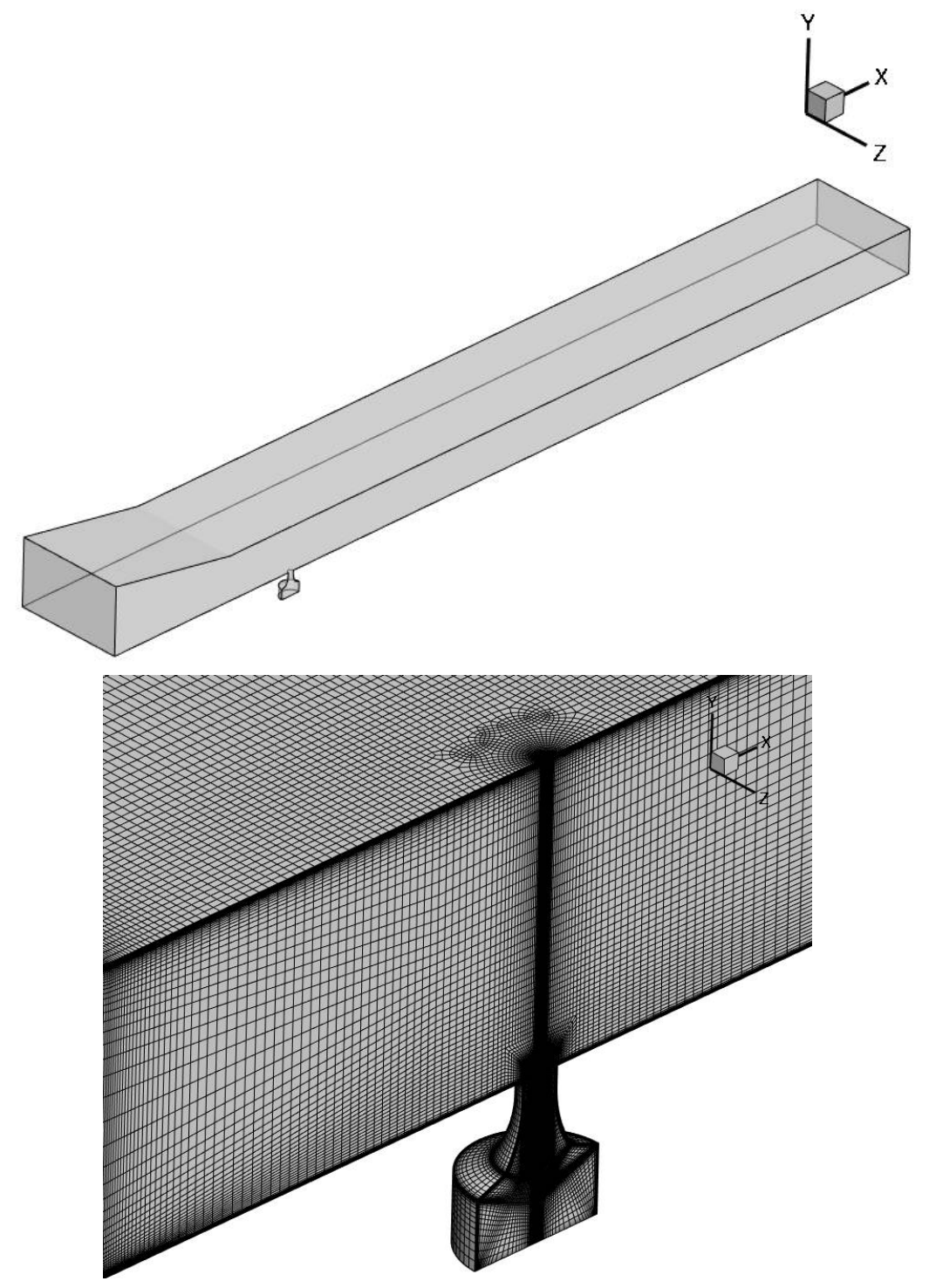

Figure 1: Grid topography

\section{Computational configuration}

Simulations of the Combined Effects Supersonic Combustor (CESCo) experiments of Gamba et al. ${ }^{7,8}$ are considered in this study. The experimental tests were conducted in a $270 \mathrm{~mm}$ long test section of a shockexpansion tube setup with a cross section of $15 \mathrm{~mm}$ (height) $\times 75 \mathrm{~mm}$ (depth). Leading into the combustion section, a $10 \mathrm{deg}$ inlet deflection angle with a capture area height of $23 \mathrm{~mm}$ is used.

The computational domain is shown in Figure 1a. The domain consists of a polyhedral unstructured mesh with a symmetric boundary along the physical center-plane of the combustor. The geometry is identical to that of the experiment, within the limit of manufacturing tolerance.

The oxidizer enters the inflow plane and fuel is introduced via an injector of diameter $2 \mathrm{~mm}$, which is located $70 \mathrm{~mm}$ downstream from the inlet leading edge. Using this setup, combustion is initiated at equivalence ratios $(\phi)$ of $0.017,0.052$, and 0.100 . To visualize combustion and heat release processes, $\mathrm{OH}^{*}$ chemiluminescence and Planar Laser-Induced Fluorescence (PLIF) was used in the experiment. Schlieren photography was used to characterize the shock train formed by the inlet geometry. Pressure measurements are available at multiple locations on the upper wall along the length of the combustor. 
The baseline computational mesh consists of 2.6 million control volumes and will be used for most of the results in this paper. A limited number of computations were performed on a fine mesh of 21 million control volumes. The mesh resolution normal to the wall was ensured to be less than 1 wall unit. A closer view of the injector region is also shown (Figure 1b). At the inflow plane, oxygen is introduced at a Mach number $M_{a}=2.8$, pressure $p_{a}=40 \mathrm{kPa}$, and temperature $T_{a}=1200 \mathrm{~K}$. For the simulations with fuel injection, three different conditions were evaluated. The plenum pressures $p_{j, o}=425 \mathrm{kPa}, 1265 \mathrm{kPa}, 2435 \mathrm{kPa}$ correspond to equivalence ratios $\phi=0.017,0.052,0.100$, respectively. Isothermal boundary conditions were applied on the viscous walls with a constant temperature of $298.15 \mathrm{~K}$.

\section{Unfueled case}

To first validate the predictions of shock-boundary layer interactions, non-reacting flow simulations (i.e. with no fuel injection) were conducted. The $10^{\circ}$ inlet ramp generates a starting oblique shock, resulting in a shock train that propagates through the entire length of the combustor. In addition, the expansion fan present at the entrance of the constant area section generates a milder alternating train of expansion and compression as seen in Figure 2. The qualitative agreement between the computed and measured shock angles appears to be extremely good. The computed pressure at the center-plane of the lower wall is compared with the experimental results in Figure 3. While the trends and correlations appear to be reasonable, an underprediction of $10 \mathrm{kPa}$ is observed. To ascertain grid convergence, a two-dimensional simulation of the symmetry plane on a mesh of 16 times finer resolution (in terms of total number of mesh elements) was performed and is included in the comparison. The results suggest that numerical discretization errors are not significant enough to explain the discrepancies.

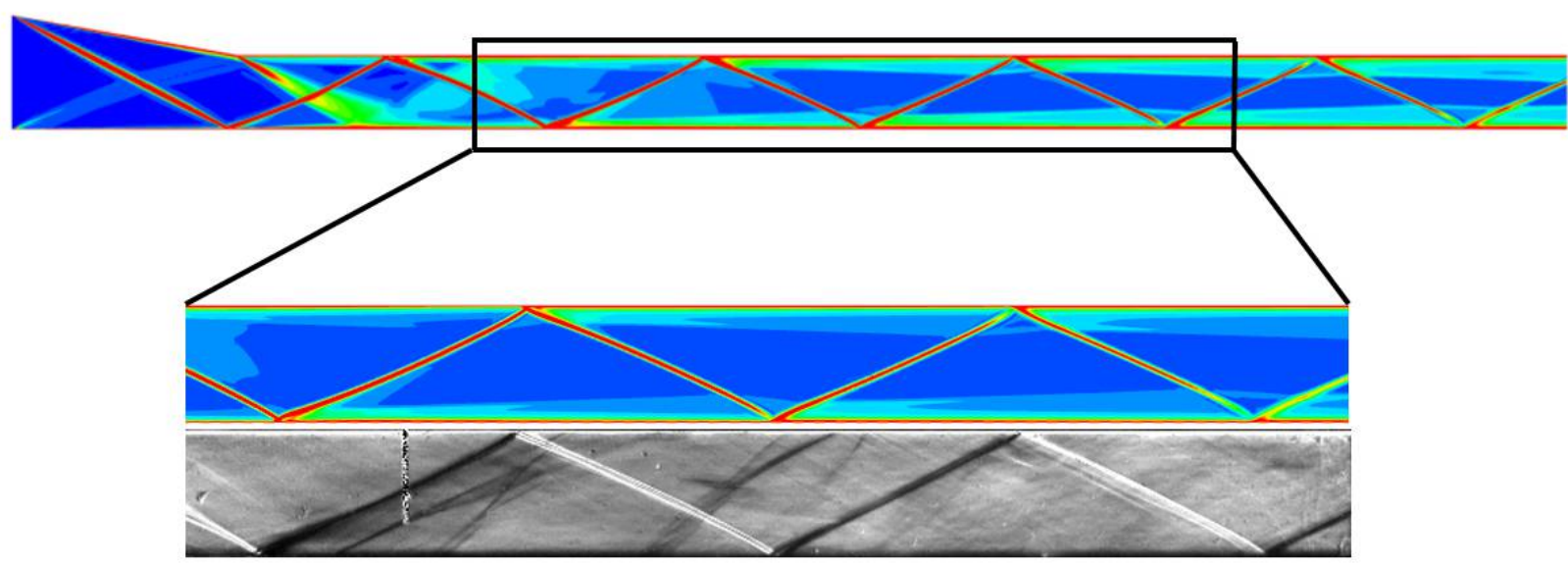

Figure 2: Density gradient contours compared with experimental Schlieren data. ${ }^{7}$

The consistent offset is a result of experimental and computational uncertainties. Of particular concern is the significant offset in the most upstream pressure tap $(x=60 \mathrm{~mm})$ just aft of the expansion wave. Since the phenomena at play constitute simple compressible flow, a potential explanation for this discrepancy could be geometric misalignment in the experimental setup or calibration errors or a mismatch of inflow conditions between the computation an experiment. Additional simulations which considered an inflow misalignment of $\pm 2^{\circ}$ were considered (Figure 4) to examine the sensitivity of the computed pressure. 


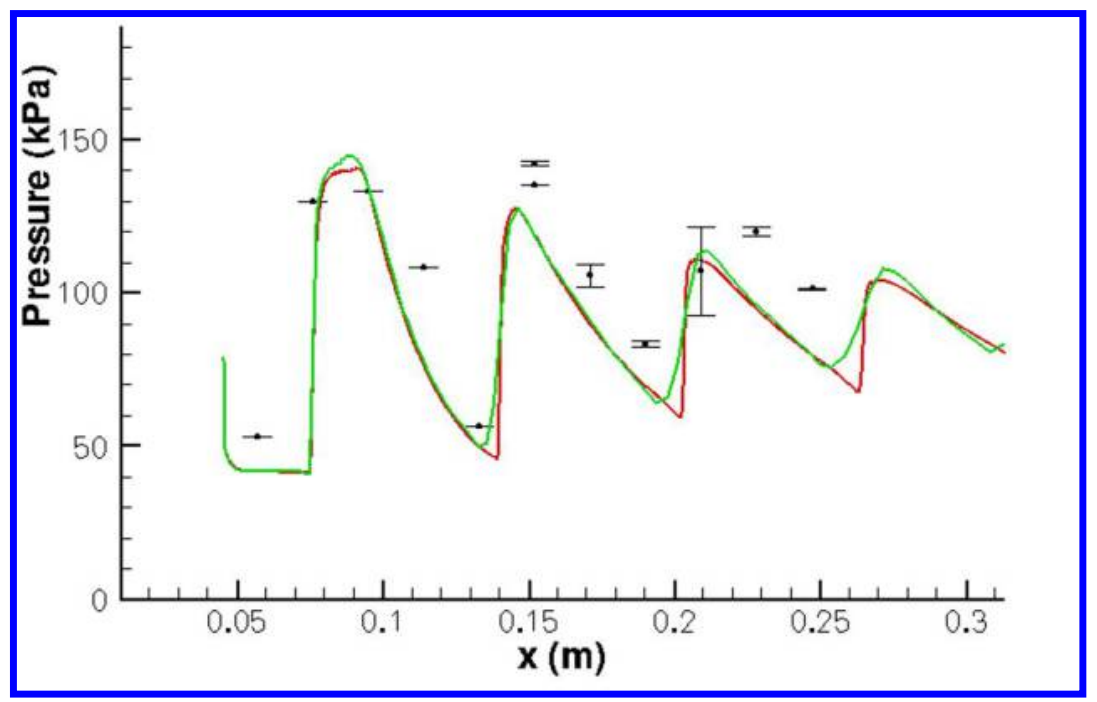

Figure 3: Pressure distribution comparison of simulation and experimental data (Experimental data provided by Gamba and Miller ${ }^{7}$ ). Green: 3D mesh; Red (2D mesh with 16x resolution).

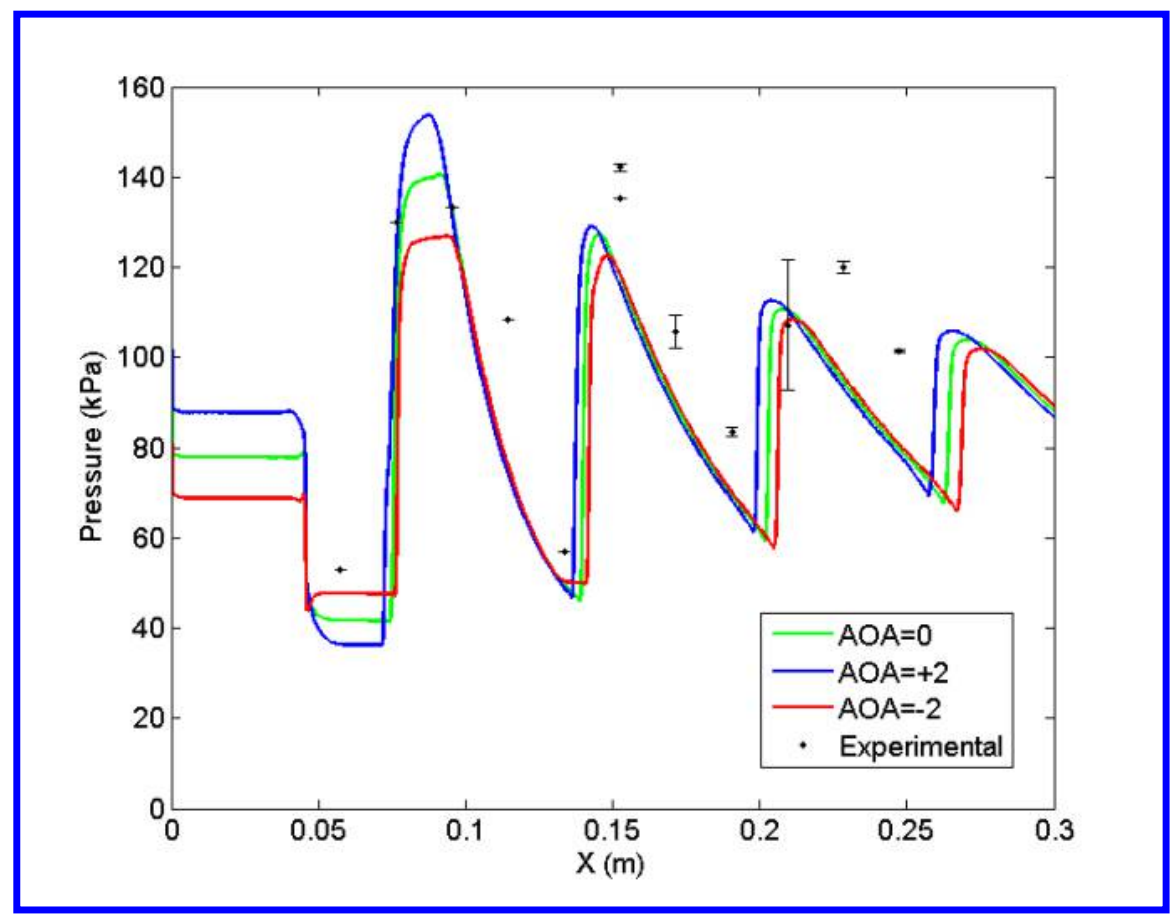

Figure 4: Comparison of computed and measured pressures along the centerline on the top wall of the combustor with misalignment angle $\alpha= \pm 2 \mathrm{deg}$.

\section{Fueled Case}

Following this validation, simulations were performed with the fuel injection corresponding to experimental equivalence ratios of $\phi=0.017,0.052$, and 0.10 . Examining the flow upstream of the injector, a small recirculation region is evident. Figure 5 shows contours of the $\mathrm{OH}$ mass fraction and the velocity field, suggesting adequate fuel and oxidizer mixing and the consequent anchoring of the flame. Immediately above the recirculation region, the intensity of the reaction appears to be diminished for all three cases. As the injection rate is increased, the anchoring region is observed to greatly increase in size. 


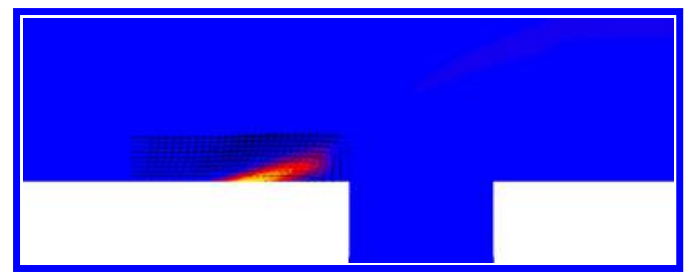

(a) $\phi=0.017$

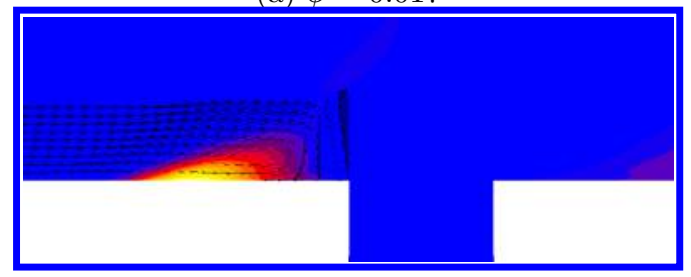

(b) $\phi=0.052$

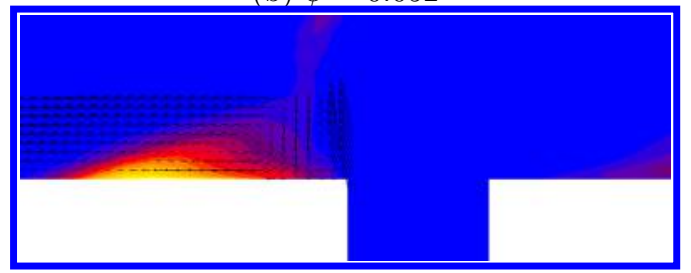

(c) $\phi=0.100$

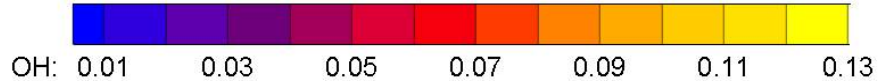

Figure 5: Comparison of recirculation regions via $\mathrm{OH}$ mass fractions.

Figure 6 shows contours of the density gradient, $\mathrm{OH}$ mass fraction and mean mixture fraction on a plane that is at a vertical distance of $2.5 \mathrm{~mm}$ from the bottom wall. The spanwise feature upstream of the injector corresponds to the first interaction of the oblique shock train with the boundary layer of the lower wall. The bow shock generated from the inlet flow is very prominent. The oblique shock train interacts with the bow shock as well as the reaction layer. This interaction is seen to interrupt the shock train, an effect that is more pronounced with increasing equivalence ratio. Examining the mixture fraction near this interaction, a lateral necking is evident, followed by an expansion of the post-shock flow. This is shown later to lead to effective flame holding. One can also examine the mixture fraction and $\mathrm{OH}$ mass fractions to assess the effect this interaction on the reaction rate. In all three cases, the shock acts to enhance the mixing and reaction downstream of the interaction.

\section{A. Planar slices and comparisons with measurements}

For additional insight, we examine the contours of the mixture fraction and the velocity divergence at the symmetry-plane (Figure 7). The bow shock system for the lowest equivalence ratio is relatively localized and more oblique in nature. The higher equivalence ratios show a much stronger bow shock that interacts with the shock train and reflects off the top wall before being disrupted by the fuel stream in a manner similar to the primary shock train. The shock system acts to suppress the penetration of the fuel flow into the core of the combustor. This is especially apparent in the low equivalence ratio case. For the larger equivalence ratios, additional interactions are visible due to the strength of the reflected bow shocks.

For lower injection rates, the fuel stream is rapidly deflected and suppressed against the bottom wall of the combustor, resulting in limited combustion due to the restricted interaction between the fuel and oxidizer streams. As the equivalence ratio is increased, the fuel stream is no longer bound to the wall.

The computed $\mathrm{OH}$ mass fraction is qualitatively compared to the OH PLIF at the center-plane of the combustor in Figure 8. While it is appreciated that the RANS represents ensemble-averaged quantities and the experiment shows instantaneous snapshots, key features of the computed reacting flow field are seen to be in excellent qualitative agreement with the experiment. In all three cases, the computation is able to track the primary reacting layer and its general patterns. While the shock train interaction is not 


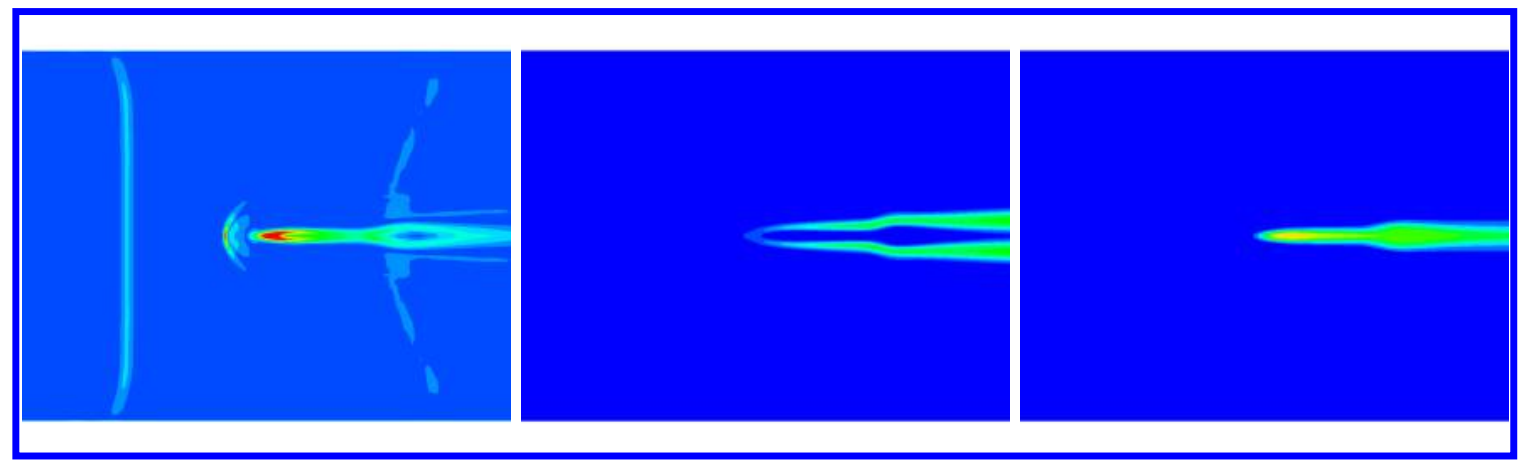

(a) $\phi=0.017$
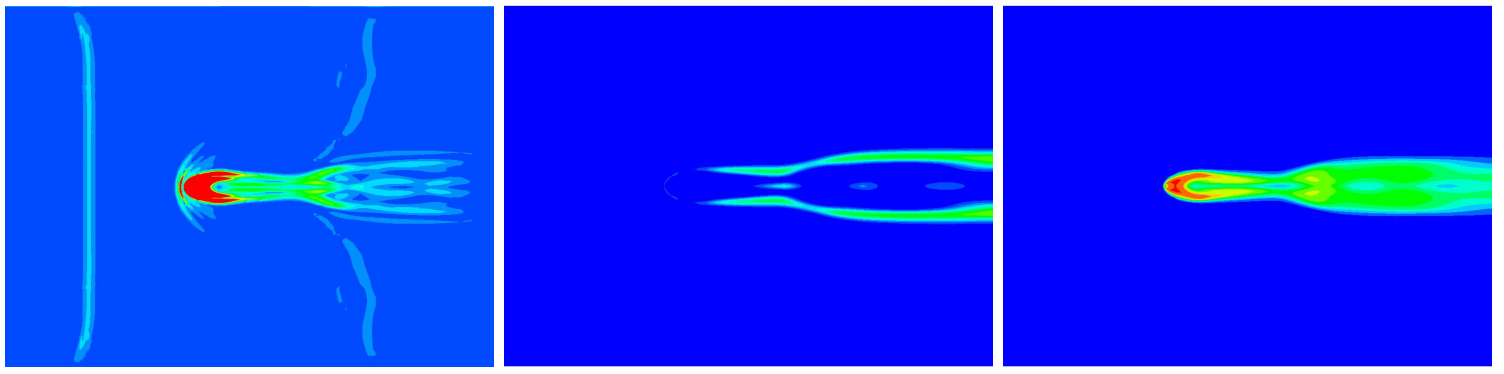

(b) $\phi=0.052$

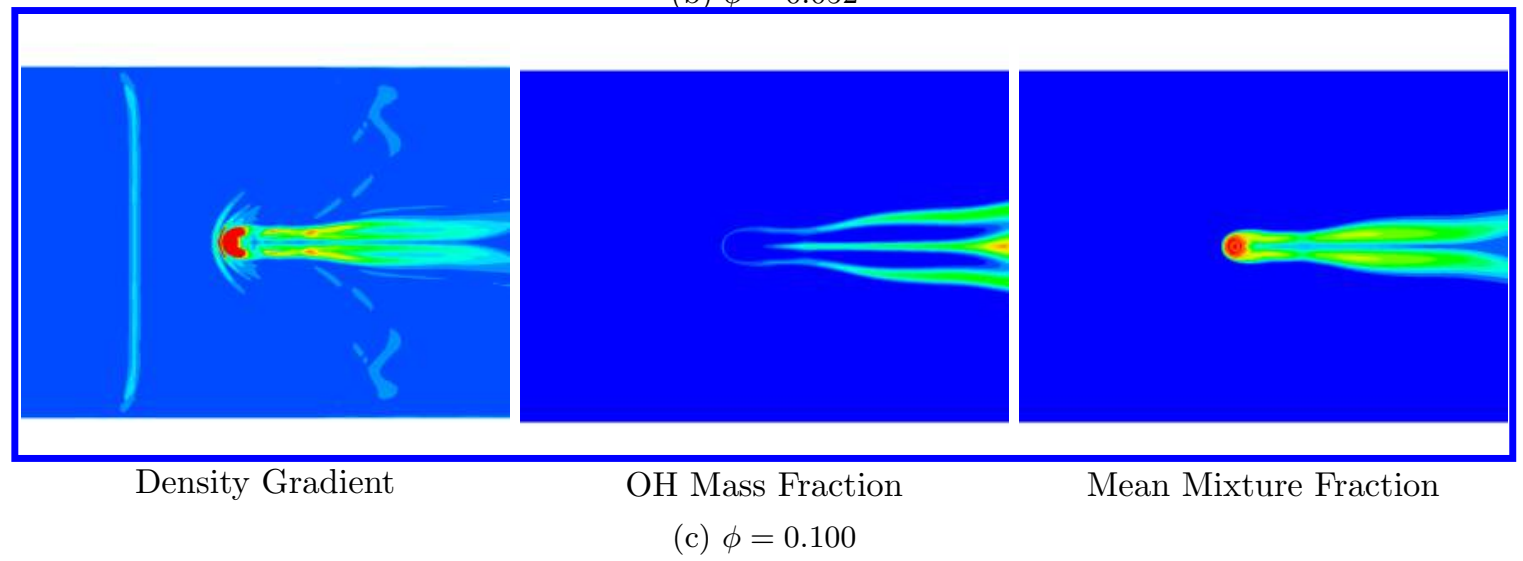

Figure 6: Top down view of injector at $\mathrm{y}=2.5 \mathrm{~mm}$ ( $16 \%$ of combustor height).

shown, the height-suppressing effect is evident. As alluded to previously, the shock train interaction acts to compress and enhance the mixing and reaction present in the layer as evidenced by the increased in $\mathrm{OH}$ in the suppressed area. This is most noticeable for $\phi=0.052$ where the reaction layer noticeably bends and thickens simultaneously.

Complementing the primary reacting layer at higher equivalence ratios, we are able to distinguish various flame-anchoring characteristics near the bottom wall. As discussed above, this additional layer occurs because the fuel flow is able to penetrate farther into the combustor. This allows mixing to occur on all sides of the fuel stream, enhancing the reaction rates. This wall-bounded reaction layer is seen to interact with the shock train in a manner similar to that of the primary reaction layer. The shock train suppresses this layer but also enhances the mixing. This is again evident in the double-ridged feature for $\phi=0.052$ as a result of interactions with the shock train and reflected bow-shock.

In Figure 9 we compare the $\mathrm{OH}$ mass fraction and heat release averaged along the span of the combustor with the $\mathrm{OH}^{*}$ chemiluminescence data for $\phi=0.052$. As noted earlier, a small reaction zone is seen to emanate from the injector, followed by a region of relatively low reaction rates. At a streamwise distance of approximately $100 \mathrm{~mm}$, which is shown earlier to be the location of the shock interaction, a marked increase 

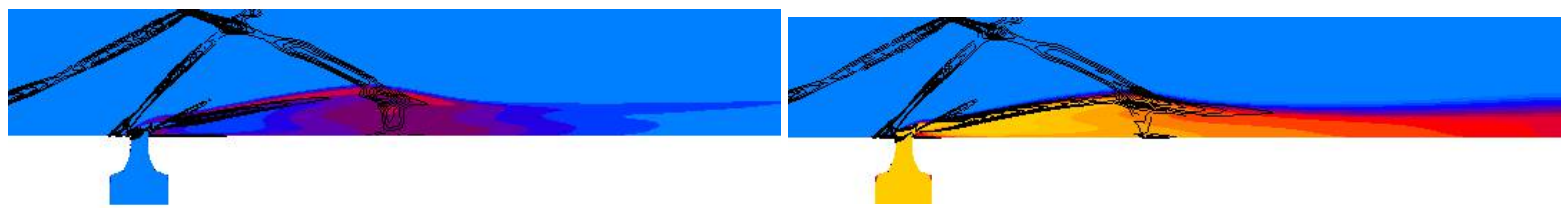

(a) $\phi=0.017$

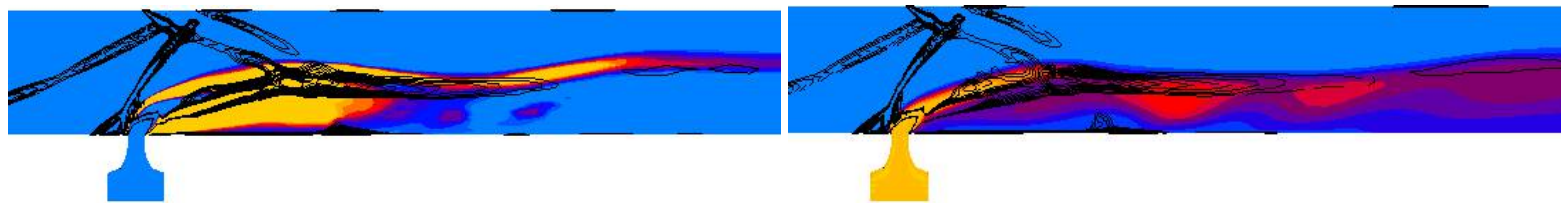

(b) $\phi=0.052$
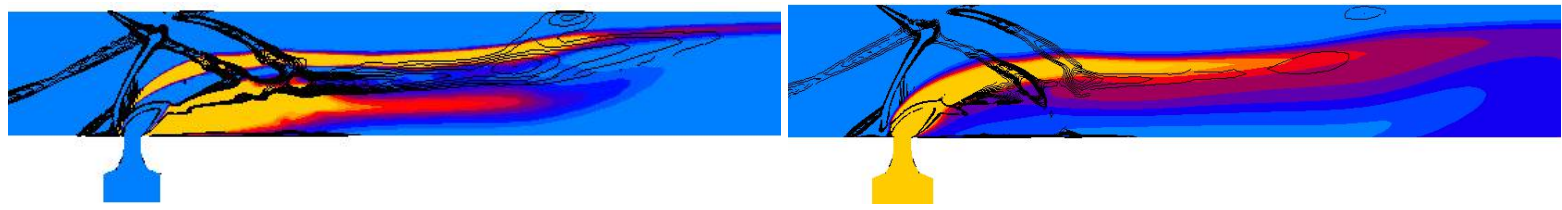

(c) $\phi=0.100$

HeatRelease: $1.0 \mathrm{E}+11 \quad 3.0 \mathrm{E}+11 \quad 5.0 \mathrm{E}+11 \quad 7.0 \mathrm{E}+11 \quad 9.0 \mathrm{E}+11 \quad 1.1 \mathrm{E}+12 \quad 1.3 \mathrm{E}+12$

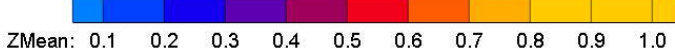

Figure 7: Centerline contours of heat release (left) and mean mixture fraction (right) overlaid with lines of constant divergence of velocity.

in $\mathrm{OH}$ radicals is generated by this improved mixing region.

Figure 10 shows the heat release integrated over each streamwise section. These results are in agreement with the qualitative assessments made previously. Following the heat release initially associated with the injection, a decrease in the sectional heat release is evident until $0.1 \mathrm{~m}$, where a secondary heat release peak is noticeable. Near this location, mixing is promoted by the shock interaction. For $\phi=0.017$, we observe that this interaction corresponds to the peak heat release (the fuel stream otherwise is confined to the wall with greatly reduced mixing).

\section{B. Comparison with pressure measurements}

In Figure 12, the computed top wall center-line pressure is compared with measurements. The impact of the heat release on the overall pressure rise is significant. In accordance with the discrepancy noted in the unfueled case, a $15 \mathrm{kPa}$ offset has been added to the measurement. While the computational and experimental results show good agreement, the sensitivity of the computed results to modeling and discretization choices remains to be assessed. The discussion below constitutes preliminary steps in this direction:

As mentioned in section II, flamelet tables were generated for a base pressure $p_{r e f}=105 \mathrm{kPa}$. Following Terrapon et al., ${ }^{21}$ the current implementation uses an adhoc scaling of the source term of the progress variable $\tilde{\dot{\omega}}_{C}=\tilde{\dot{\omega}}_{0 C} \frac{\bar{p}^{2}}{p_{r e f}^{2}}$ to adjust for pressure variations. To ascertain the sensitivity of the overall results to the base pressures, a variety of pressure tables were generated for reference pressure ranging from $50 \mathrm{kPa}$ to $200 \mathrm{kPa}$. The centerline wall pressures from some of these simulations are shown in Figure 13a. In general, pressure variations do not significantly affect the results, suggesting that discrepancies could be due to other limiting factors in flamelet and turbulence modeling.

To assess the effect of the grid resolution on the simulation results, a uniform refinement was applied to the baseline grid. Each control volume was further divided uniformly in all three directions, resulting in a total of 21 Million mesh elements. The comparison in Figure 13b shows a limited sensitivity to mesh refinement. 


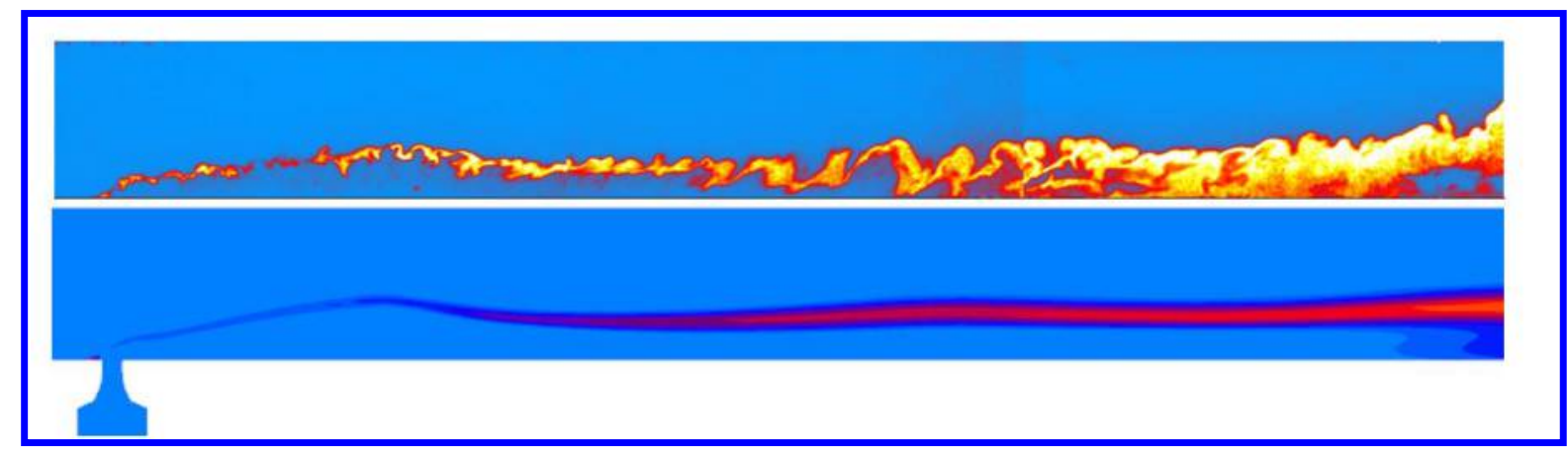

(a) $\phi=0.017$

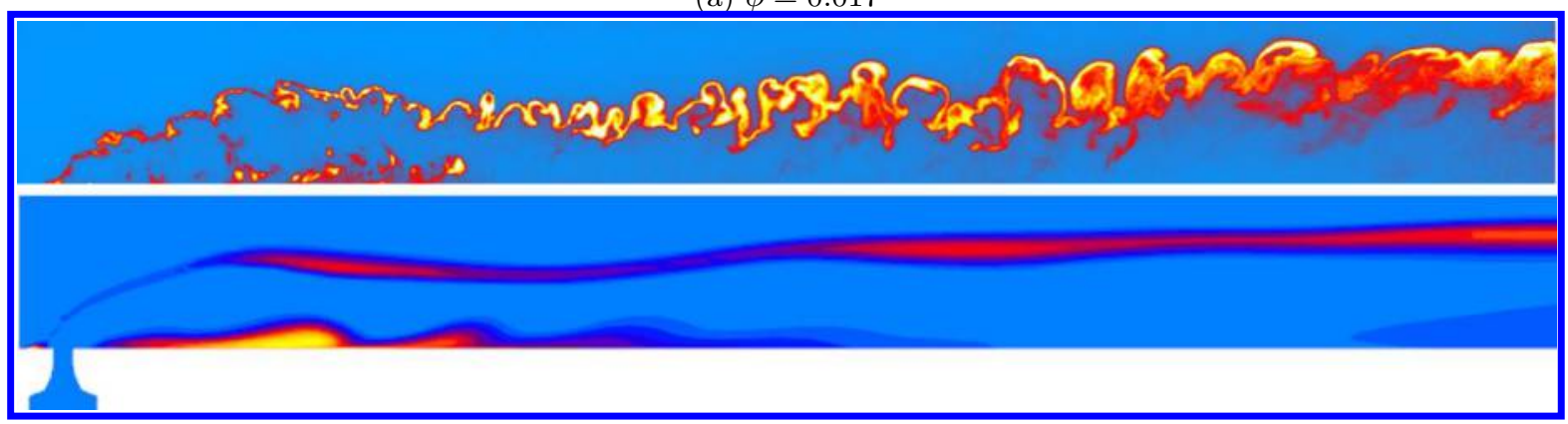

(b) $\phi=0.052$

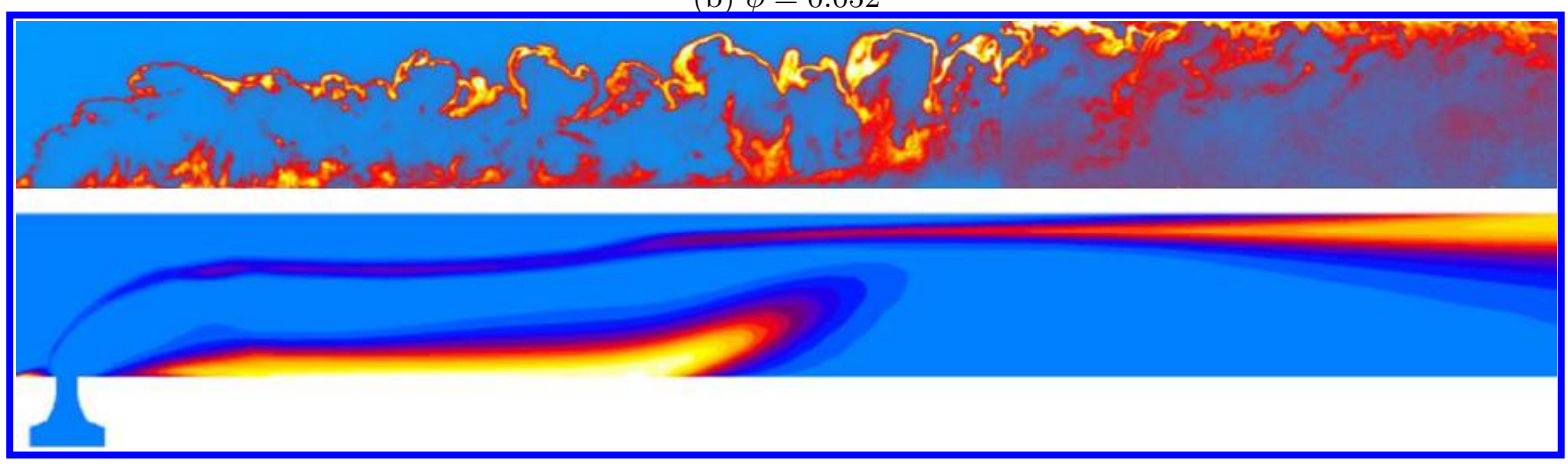

(c) $\phi=0.100$

Figure 8: In each set, the top figure corresponds to the measured (instantaneous) OH PLIF and the bottom figure corresponds to the computed (ensemble-averaged) $\mathrm{OH}$ mass fraction. (Experimental data provided by Gamba and Miller).

\section{Conclusions}

In this work, the flamelet progress variable approach (FPVA) was applied to a model Scramjet combustor in a RANS context. The flow configuration consists of a shock train interacting with the injection of a single jet. This setup represents an intermediate level of complexity between a simple jet-in-crossflow and a full combustor and provides a convenient framework to analyze relevant flow and combustion processes in the presence of confinement effects.

Computations of the unfueled (non-reacting) configuration showed good qualitative agreement of shock train features and quantitative agreement in pressure (with an offset of $10-15 \mathrm{kPa}$ ) with measurements. Reacting flow simulations were conducted at a variety of equivalence ratios. The shock-train / combustion layer interaction resulted in the enhancement of combustion and flame anchoring. The penetration of the fuel stream towards the center of the channel was shown to directly control mixing. At higher equivalence ratios, the bow shock further enhanced the reaction rate. The impact of the shock interactions on the heat release was examined. Key flow features were found to be in excellent qualitative agreement with planar laser induced fluorescence and chemiluminescence imaging. 


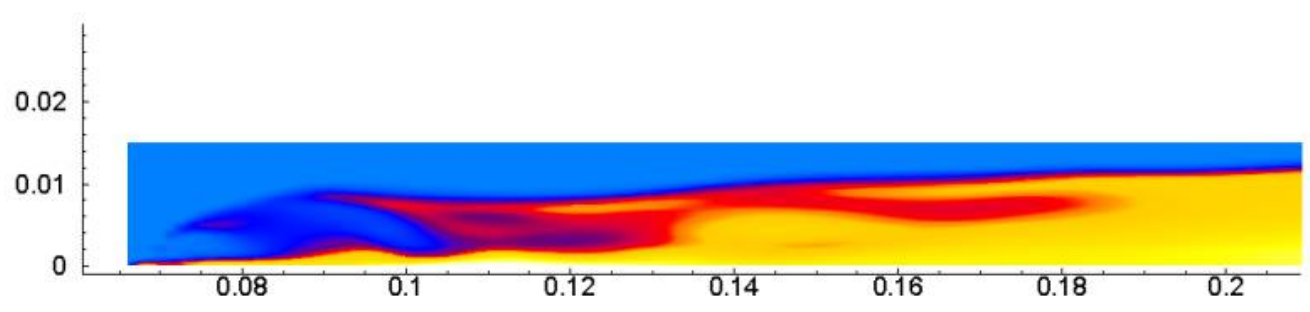

(a) LOS averaged $\mathrm{OH}$ mass fraction contours (axis in $\mathrm{m}$ )

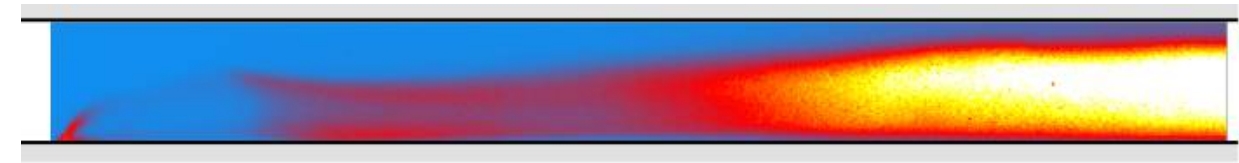

(b) $\mathrm{OH}^{*}$ Chemiluminescence with exposure time of $100 \mu \mathrm{s}^{7}$

Figure 9: Comparison of line of sight at $\phi=0.052$.

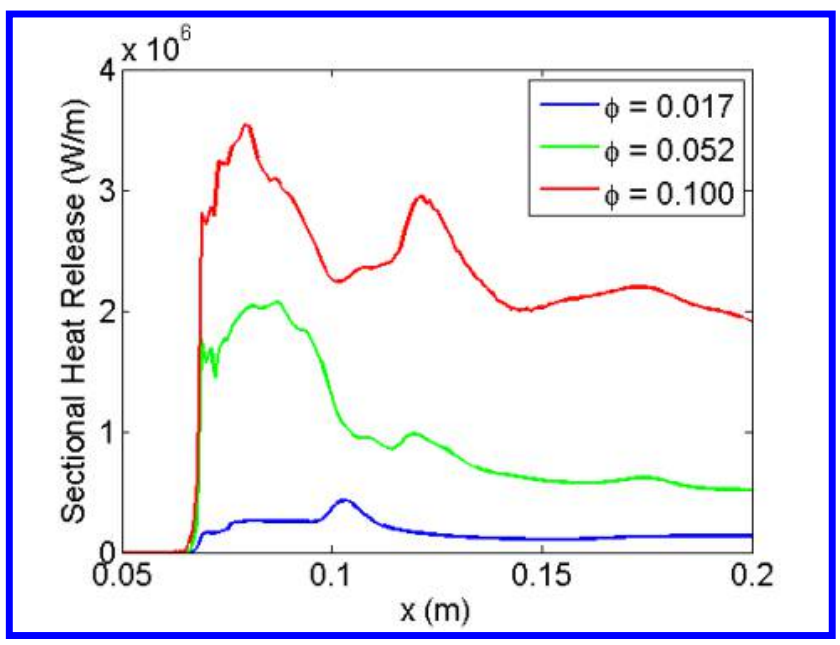

Figure 10: Sectional heat release along combustor length.

Aside from a constant offset of $10-15 \mathrm{kPa}$, a very good degree of correlation was observed between the computed and measured wall pressures. The results were confirmed to be weakly sensitive to change in flamelet reference pressure and grid refinement.

This work represents the first steps toward a deeper investigation into quantifying uncertainties in RANS computations of Scramjet systems. A broader goal is to develop effective reduced-order models to characterize unstart and to integrate the tools within a design framework. Ongoing work is dedicated towards assessing the impact of model-form errors on the heat release using data-driven techniques. ${ }^{6}$

\section{Acknowledgments}

This work was partially supported by the Department of Energy Predictive Science Academic Alliance Program (Grant DE-FC52-08NA28614). The authors acknowledge Mirko Gamba (University of Michigan) and Victor Miller (Stanford University) for supplying the experimental results, including some that are provisional in nature.

\section{References}

${ }^{1} \mathrm{R}$ S Barlow and J H Frank. Effects of Turbulence on Species Mass Fractions in Methane/air Jet Flames. In TwentySeventh Symposium (International) on Combustion/The Combustion Institute, pages 1087-1095, 1998. 


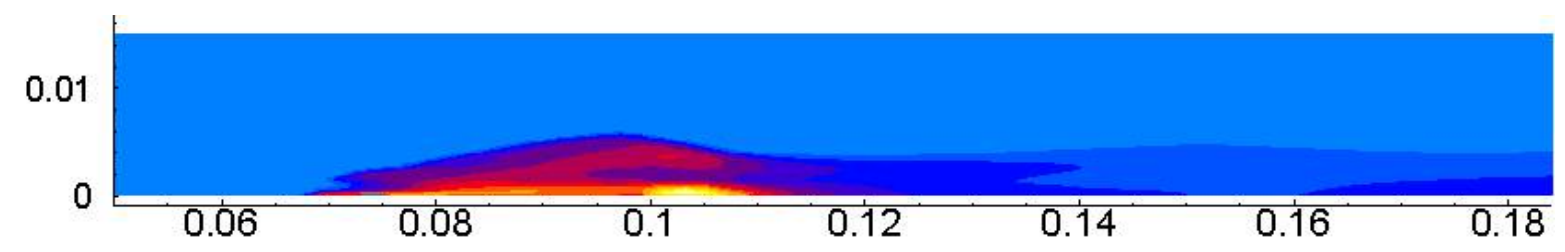

(a) $\phi=0.017$

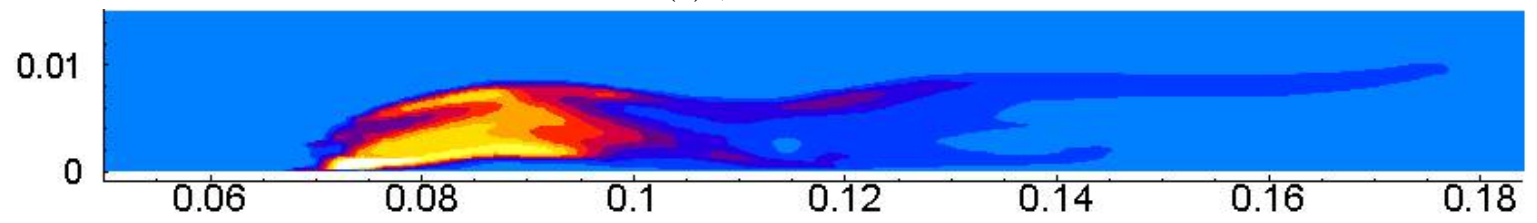

(b) $\phi=0.052$

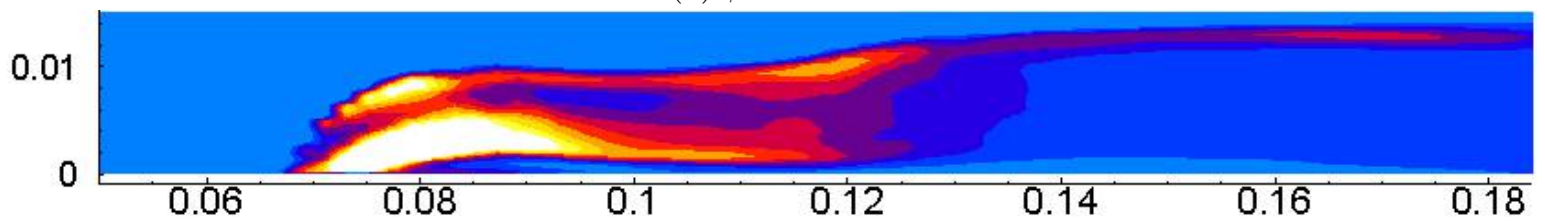

(c) $\phi=0.100$

Spanwise-averaged heat release.

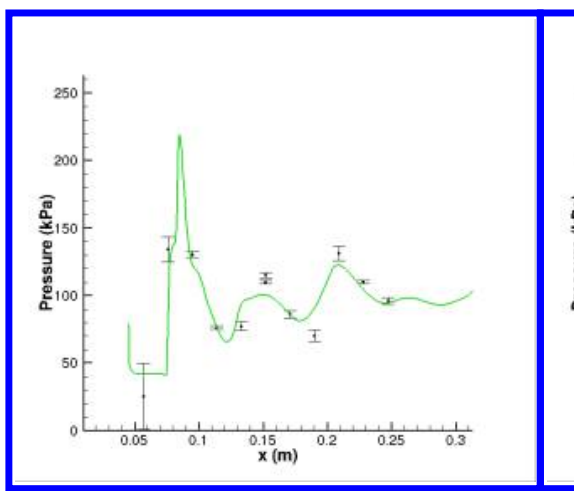

a) $\phi=0.017$

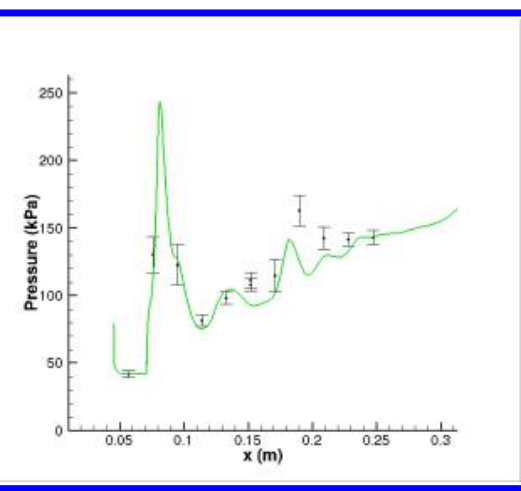

b) $\phi=0.052$

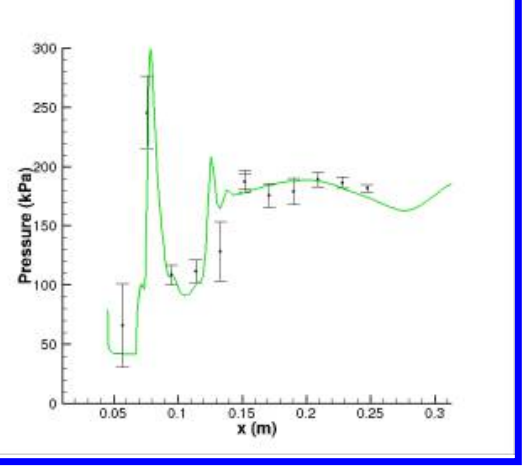

c) $\phi=0.100$

Figure 12: Centerline pressure (shifted) along the top wall of the combustor. (Experimental data provided by Gamba and Miller).

${ }^{2} \mathrm{R}$ W Bilger, S B Pope, K N C Bray, and J F Driscoll. Paradigms in turbulent combustion research. In Proceedings of the Combustion Institute, volume 30, pages 21-42, 2006.

${ }^{3}$ W Kendal Bushe, Helfried Steiner, W Kendal Bushe, and Helfried Steiner. Conditional moment closure for large eddy simulation of nonpremixed turbulent reacting flows Conditional moment closure for large eddy simulation of nonpremixed turbulent reacting flows. Physics of Fluids, 1896(May 2016):1896-906, 1999.

${ }^{4}$ Pratik Donde, Heeseok Koo, and Venkat Raman. A multivariate quadrature based moment method for LES based modeling of supersonic combustion. Journal of Computational Physics, 231(17):5805-5821, 2012.

${ }^{5}$ Karthik Duraisamy and Juan Alonso. Adjoint Based Techniques for Uncertainty Quantification in Turbulent Flows with Combustion. (June):1-16, 2012.

${ }^{6}$ Karthik Duraisamy Eric J. Parish. A paradigm for data-driven predictive modeling using field inversion and machine learning. Journal of Computational Physics, 305:758-774, 2016.

${ }^{7}$ Mirko Gamba, V A Miller, M G Mungal, and R K Hanson. Combustion Characteristics of an Inlet/Supersonic Combustor Model. 50th AIAA Aerospace Sciences Meeting including the New Horizons Forum and Aerospace Exposition, (AIAA 20120612), 2012.

${ }^{8}$ Mirko Gamba, Victor A Miller, M Godfrey Mungal, and Ronald K Hanson. Ignition and Flame Structure in a Compact Inlet/Scramjet Combustor Model. In 17th AIAA International Space Planes and Hypersonic Systems and Technologies Conference, number April, 2011.

${ }^{9}$ Ivan Bermejo-Moreno Julien Bodart Sebastian Karl Ronan Vicquelin Johan Larsson, Stuart Laurence. Incipient thermal choking and stable shock-train formation in the heat-release region of a scramjet combustor. part ii: Large eddy simulations. Combustion and Flame, 162:907-920, 2015. 


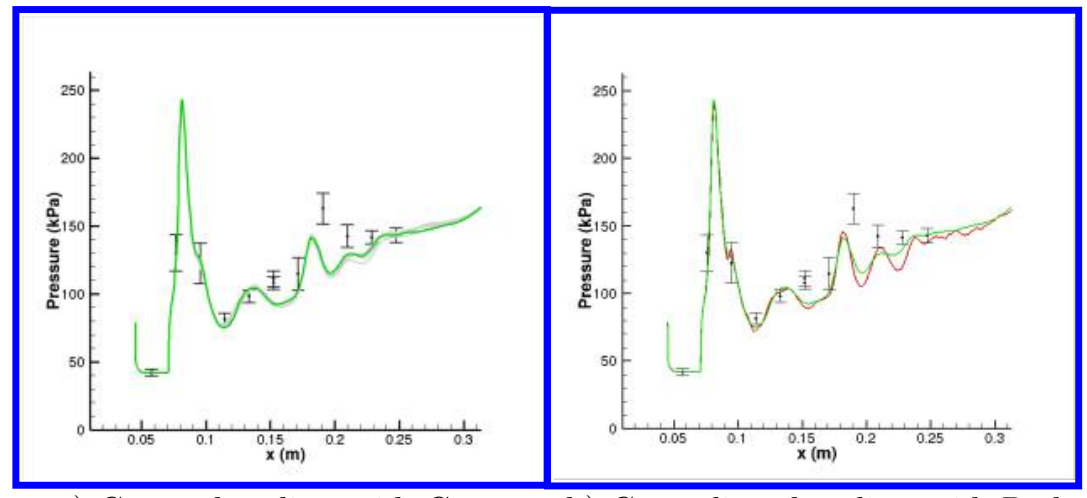

a) Green: baseline grid; Grey: b) Green line: baseline grid; Red pressure variation. line: finer grid with $8 \mathrm{x}$ resolution.

Figure 13: Sensitivity of top wall pressure to reference pressure in flamelet table and mesh refinement: $\phi=0.052$. (Experimental data provided by Gamba and Miller)

\footnotetext{
${ }^{10}$ Sebastian Karl, Jan Martinez Schramm, Klaus Hannemann, and Stuart Laurence. CFD Analysis of Unstart Characteristics of the HyShot-II Scramjet Configuration in the HEG Shock Tunnel. 17th AIAA International Space Planes and Hypersonic Systems and Technologies Conference, (April):1-11, 2011.

${ }^{11} \mathrm{~A}$ Y Klimenko and R W Bilger. Conditional moment closure for turbulent combustion. Progress in Energy and Combustion Science, 25(1999):595-687, 2006.

${ }^{12}$ F. R Menter. Two-equation eddy-viscosity turbulence models for engineering applications. AIAA Journal, 32:1598-1605, 1994.

${ }^{13}$ Rene Pecnik, VE Terrapon, Frank Ham, and Gianluca Iaccarino. Full system scramjet simulation. Annual Research Briefs, pages 33-45, 2009.

${ }^{14}$ René Pecnik, Vincent E. Terrapon, Frank Ham, Gianluca Iaccarino, and Heinz Pitsch. Reynolds-Averaged Navier-Stokes Simulations of the HyShot II Scramjet. AIAA Journal, 50(8):1717-1732, 2012.

${ }^{15} \mathrm{~N}$ Peters. A spectral closure for premixed turbulent combustion in the flamelet regime. Journal of Fluid Mechanics, 242:611-29, 1992.

${ }^{16}$ Charles D. Pierce and Parviz Moin. Progress-variable approach for large-eddy simulation of non-premixed turbulent combustion. Journal of Fluid Mechanics, 504:73-97, 2003.

${ }^{17}$ H. Pitsch. Flamemaster, A C++ Computer Program for 0D and 1D Laminar Flame Calulations.

${ }^{18}$ Heinz Pitsch. Large-Eddy Simulation of Turbulent Combustion. Annual Review of Fluid Mechanics, 38(1):-482, 2006.

${ }^{19}$ Amirreza Saghafian, Vincent E Terrapon, Frank Ham, and Heinz Pitsch. An efficient flamelet-based combustion model for supersonic flows. In Paper No. AIAA-2011-2267, 17th AIAA International Space Planes and Hypersonic Systems and Technologies Conference, April, pages 11-14, 2011.

${ }^{20}$ Amirreza Saghafian, Vincent E. Terrapon, and Heinz Pitsch. An efficient flamelet-based combustion model for compressible flows. Combustion and Flame, 162(3):652-667, 2015.

${ }^{21}$ By V E Terrapon, F Ham, R Pecnik, and H Pitsch. A flamelet-based model for supersonic combustion. Ctrstanfordedu, pages $47-58,2009$.

${ }^{22}$ Julian M Tishkoff, J. P. Drummond, T. Edwards, and A. S. Nejad. Future directions of supersonic combustion research - Air Force / NASA workshop on supersonic combustion Future Directions of Supersonic Combustion Research : Air Force / NASA Workshop on Supersonic Combustion. In AIAA, Aerospace Sciences Meeting \{ 6$\}$ Exhibit, number January, 1997.

${ }^{23} \mathrm{~V}$ Venkatakrishnan. Convergence to Steady State Solutions of the Euler Equations on Unstructured Grids with Limiters, 1995.

${ }^{24}$ D. C. Wilcox. Turbulence modeling for CFD. DCW industries., La Canada, CA, 2006.
} 